\title{
Serum uric acid and the incidence of CKD and hypertension
}

\author{
Satoru Kuriyama ${ }^{2} \cdot$ Yukio Maruyama $^{2}$ - Shinichiro Nishio $^{2}$ - Yasuhito Takahashi ${ }^{2}$. \\ Satoshi Kidoguchi $^{2}$. Chisa Kobayashi ${ }^{2}$ - Daisuke Takahashi ${ }^{2} \cdot$ Naoki Sugano $^{2}$. \\ Tatsuo Hosoya ${ }^{1} \cdot$ Takashi Yokoo $^{2}$
}

Received: 8 December 2014/Accepted: 29 April 2015/Published online: 13 May 2015

(c) The Author(s) 2015. This article is published with open access at Springerlink.com

\begin{abstract}
Background Uric acid (UA) levels correlate positively with the prevalence of chronic kidney disease (CKD) and/ or hypertension. We tested the hypothesis that UA may also have a link to a new incidence of CKD and hypertension.

Methods Study design is a cohort study and the predictor is UA levels. Of the 15,470 screened cases, 8223 participants without CKD were eligible for the analysis of the incidence of CKD. Among these CKD candidates, 7569 participants were eligible for the analysis of the new development of hypertension. The observation period was 4 years.

Results Relationship of UA with new cases of CKD. Higher UA levels had a closer association with the new development of CKD; $1.1 \%$ (UA $<5 \mathrm{mg} / \mathrm{dL}$ ), $1.5 \%$ $(5.0-5.9 \mathrm{mg} / \mathrm{dL}), \quad 1.7 \% \quad(6.0-6.9 \mathrm{mg} / \mathrm{dL}), \quad$ and $3.4 \%$ ( $\geqq 7 \mathrm{mg} / \mathrm{dL}$ ), respectively $(p<0.001$ by the Chi-square test). Cox proportional hazard analysis showed that the estimates of the CKD development were eGFR [Hazard Ratio (HR) 0.816, $95 \%$ confidence intervals (CI) 0.791-0.840] and male gender (HR 0.562, $95 \%$ CI $0.322-0.982$ ). UA levels and new development of hypertension. Higher UA levels had a closer association with the new development of hypertension; $5.0 \%$ (UA $<5 \mathrm{mg}$ /
\end{abstract}

Satoru Kuriyama

kuriyamas218@yahoo.co.jp

1 Department of Pathophysiology and Therapy in Chronic Kidney Disease, Jikei University School of Medicine, Tokyo, Japan

2 Division of Nephrology and Hypertension, Department of Internal Medicine, Jikei University School of Medicine, 3-258, Nishi-shinbashi, Minato-ku, Tokyo 105-8461, Japan
$\mathrm{dL}), 8.9 \%(5.0-5.9 \mathrm{mg} / \mathrm{dL}), 10.6 \%(6.0-6.9 \mathrm{mg} / \mathrm{dL})$, and $11.8 \%(\geqq 7 \mathrm{mg} / \mathrm{dL})$, respectively $(p<0.001$ by the Chisquare test). Cox proportional hazard analysis showed that the estimates of the hypertension development were BMI (HR 1.190, $95 \%$ CI 1.155-1.226), age (HR 1.021, $95 \%$ CI 1.010-1.032), HDL-cholesterol (HR 1.013, $95 \%$ CI 1.007-1.019), male gender (HR 1.791, $95 \%$ CI 1.338-2.395), UA level (HR 1.112, $95 \%$ CI 1.024-1.207), and eGFR (HR 1008, 95 \% CI 1.002-1.013). Furthermore, the logistic analysis showed that the odds ratio (OR) to estimate hypertension in the high UA group (UA $\geqq 7 \mathrm{mg} /$ $\mathrm{dL}$; OR 1.33, $95 \%$ CI 1.01-1.80) was greater than that in the low UA group (UA $<5 \mathrm{mg} / \mathrm{dL}$ ). Kaplan-Meier analysis also confirmed the finding that the higher the UA levels the greater the hypertension development $(p<0.001$ by the Log-rank test and Cox proportional hazard analysis). Conclusion High UA levels are associated with the new development of hypertension, but not with the incidence of CKD.

Keywords Uric acid - CKD - Hypertension - Estimated glomerular filtration rate

\section{Introduction}

Previous studies showed that circulating high uric acid (UA) levels were associated with increased prevalence of hypertension and a high risk status of cardiovascular complications which frequently leads to poor patient prognosis [1-6]. Increased UA levels may also play a pivotal role in the progression of chronic kidney disease (CKD) such as chronic glomerulonephritis [7], diabetic nephropathy [5, 8], end stage renal failure [7, 8], and gouty kidney [9]. These data are suggestive of UA as a precipitating factor in the 
progression of CKD and/or hypertension. The potential mechanisms to account for these associations may be diverse; i.e., endothelial dysfunction, the activation of the intrarenal renin-angiotensin system, a vascular smooth muscle cell proliferation, the increased synthesis of interleukin-6, insulin resistance, and impaired endothelial nitric oxide productions [10]. Regardless of these affirmative reports, the relationship between UA levels and CKD or hypertension is not always consistent. In fact, some other studies performed in other countries did not support such an association between kidney dysfunction and hyperuricemia [11-14]. At this point, therefore, one can conclude the role of hyperuricemia in the CKD progression and hypertension in humans is still a matter of controversy. Regarding the relationship of early onset with UA level, evidences are much more scant. Thus, further studies to investigate whether UA can trigger the early onset of CKD and/or hypertension are needed to address this question.

In the present study, with the above-mentioned background, we carried out a longitudinal retrospective survey to look into the associations of UA level with the development of hypertension and the new onset of CKD in a screened population at large. We applied strict exclusion criteria in order to choose eligible participants who did not have a concurrent or a past history of CKD and/or hypertension.

\section{Subjects and methods}

\section{Study population and design}

The study design is a retrospective population-based cohort of Japanese office workers aged 25-60 years, living in the vicinity of Tokyo. The original number of the participants was 15,470 who had an annual medical check-up every year from 2008 to 2012. They were seen at least once a year by well-trained physicians or public nurses.

The basic criteria excludes individuals receiving medications for diabetes, hyperuricemia, hypertension, and dyslipidemia throughout the follow-up period of 4 years; those having insufficient data; those with past history of incident major cardiovascular events (MACE) such as cerebral apoplexy or myocardial infarction; those with any disease requiring hospitalization; those with current cancer or other life-threatening diseases; those with current pregnancy, and those with estimated glomerular filtration rate (eGFR) less than $60 \mathrm{~mL} / \mathrm{min} / 1.73 \mathrm{~m}^{2}$. The present and past history of proteinuria was also another rule-out factor. This exclusion process finally leaves 8223 for the analysis of the new onset of CKD. Finally, the additional application of exclusion criterion of a blood pressure (BP) rise to systolic of $140 \mathrm{mmHg}$ or greater, and diastolic of $90 \mathrm{mmHg}$ or greater at the beginning leaves 7569 individuals for the analyses of the new development of hypertension.

\section{Primary predictor and outcome}

The primary predictor was the level of UA. UA levels were divided into 4 groups (Group 1: UA $<5.0 \mathrm{mg} / \mathrm{dL}$, Group 2: UA $5.0-5.9 \mathrm{mg} / \mathrm{dL}$, Group 3: UA 6.0-6.9 mg/dL, Group 4: $\mathrm{UA} \geqq 7.0 \mathrm{mg} / \mathrm{dL}$ ) and were evaluated in conjunction with newly developed CKD and hypertension. The new incidence of CKD was defined as a decline in eGFR to less than $60 \mathrm{~mL} / \mathrm{min} / 1.73 \mathrm{~m}^{2}$ calculated each year. Similarly, the new onset of hypertension was defined as a rise of systolic blood pressure (SBP) to $140 \mathrm{mmHg}$ or greater, and/or diastolic blood pressure (DBP) of $90 \mathrm{mmHg}$ or greater evaluated at each year. Renal function expressed as eGFR for Japanese was calculated based on the equation; $\mathrm{eGFR}=194 \times \mathrm{Cr}^{-1.094} \times \mathrm{Age}^{-0.287}($ if women $\times 0.739)$, reported elsewhere [15].

\section{Other variables}

Body mass index (BMI) was calculated based on the equation; $\mathrm{BMI}=$ Body weight $(\mathrm{BW}) \times 1 /($ Body Height $)$ squared. Laboratory tests were carried out after an 8-to 12-h fast. Measurements were made on serum creatinine (Cr) concentration, serum uric acid (UA) concentration, blood urea nitrogen (BUN), electrolytes, and lipid profiles including total cholesterol (TC), triglycerides (TG), highdensity lipoprotein cholesterol (HDLC), low-density lipoprotein cholesterol (LDLC), HbA1c, and plasma glucose (PG). Laboratory tests were performed using the Toshiba auto-analyzer (TBA-80 FR neo, Tokyo, Japan). Urinalysis was performed for qualitative measurement of urinary protein. Quantitative measurement of daily urinary protein excretion was not available. The value of $\mathrm{HbAlc}$ was measured as a unit of JDS (Japan Diabetes Society). Following the worldwide recommendations, all of the HbA1c values were expressed as a unit of NGSP (National glycohemoglobin standardization program) based on the equation; NGSP $(\%)=$ JSD $\times 1.02+0.25(\%)$.

Blood pressure (BP) was measured in a sitting position during a morning visit (fasting, 9-11 AM), after 5 min of rest in the supine position by an automatic self-measuring device equipped with a $47 \times 13 \mathrm{~cm}$ cuff and $24 \times 13 \mathrm{~cm}$ bladder to avoid so-called "white coat hypertension" and/ or "cuff hypertension".

\section{Ethical considerations}

The present study was conducted in accordance with "Recommendations on the Establishment of Animal 
Experimental Guidelines" approved at the 80th General Assembly of the Japanese Science Council in 1980, and the principles set out in the Declaration of Helsinki 1964 as modified by subsequent revisions.

The study protocol design was a retrospective screened cohort. This epidemiological survey was submitted to the Institutional Review Board (IRB)/Ethics Committee of the Jikei University School of Medicine. After the deliberation the protocol was approved by the ethical committee of the University with the clinical trial number 25-203 (7338).

\section{Statistical analysis}

Uric acid was analyzed by dividing the distribution into 4 groups. Cross-sectional associations of baseline demographics and risk factors with UA groups were performed using Chi-square analysis for discrete variables, analysis of variance (ANOVA) for continuous variables. $p$ values were calculated for linear trend across the 4 UA groups. Cox proportional hazard analysis was used to estimate adjusted hazard ratio (HR) and associated $95 \%$ confidence intervals (CI) for either CKD onset or hypertension development. In the longitudinal analysis, we examined the association of new incident CKD and new onset of hypertension according to baseline UA levels. For these dichotomous outcomes, logistic regression analysis was used to estimate adjusted odds ratio (OR) and associated $95 \% \mathrm{CI}$ in relation to 4 UA groups. UA was modeled as both a linear variable per $1 \mathrm{mg} / \mathrm{dL}$ increase.
Variables significant in univariate analyses were considered to be potential confounders of UA in multivariate models. The final model was adjusted for age, gender, BMI, baseline serum $\mathrm{Cr}$ concentration, SBP and DBP, HbA1c and lipid levels. The final variables were chosen on the basis of biological plausibility at the doctor's discretion. After obtaining the statistical significance as the estimate in the multivariate analysis, univariate analysis to estimate time-to-disease-free curves in relation to the UA levels was performed using Kaplan-Meier method and compared with stratified Log-rank tests. In addition, Cox proportional hazard analysis was employed to evaluate the statistical difference among the 4 UA groups.

Statistical analyses were carried out with Stat Flex version 6.0 (Artec Ltd. Co., Osaka, Japan). Data are presented as the mean \pm standard deviation (SD), unless otherwise indicated. $p<0.05$ is considered statistically significant. Confidence intervals (CI) are expressed as $95 \%$ CI.

\section{Results}

\section{CKD analysis}

\section{Baseline Characteristics}

The study cohort had an average age of $39 \pm 10$ years, an eGFR of $91.2 \pm 17.6 \mathrm{~mL} / \mathrm{min} / 1.73 \mathrm{~m}^{2}$, a UA level of

Table 1 Demographics of subjects for CKD onset

\begin{tabular}{|c|c|c|c|c|c|c|}
\hline & \multicolumn{6}{|c|}{ Uric acid groups (mg/dL) } \\
\hline & Overall & $<5.0$ & $5.0-5.9$ & $6.0-6.9$ & $\geqq 7.0$ & $p$ \\
\hline$N$ & 8223 & 2631 & 2418 & 2118 & 1056 & \\
\hline Age (years) & $39 \pm 10$ & $37 \pm 9$ & $39 \pm 10$ & $40 \pm 10$ & $41 \pm 9$ & $<0.001$ \\
\hline Men $(\%)$ & 77.7 & 42.2 & 89.6 & 97.9 & 98.8 & $<0.001$ \\
\hline Hypertension (\%) & 7.7 & 3.8 & 7.1 & 10.6 & 15.1 & $<0.001$ \\
\hline BMI $\left(\mathrm{Kg} / \mathrm{m}^{2}\right)$ & $22 \pm 3$ & $21 \pm 3$ & $22 \pm 3$ & $23 \pm 3$ & $24 \pm 3$ & $<0.001$ \\
\hline Waist circumference $(\mathrm{cm})$ & $78.6 \pm 8.8$ & $74.1 \pm 8.2$ & $78.6 \pm 7.9$ & $81.1 \pm 7.9$ & $84.2 \pm 8.6$ & $<0.001$ \\
\hline $\mathrm{SBP}(\mathrm{mmHg})$ & $118 \pm 13$ & $113 \pm 12$ & $118 \pm 12$ & $120 \pm 13$ & $123 \pm 14$ & $<0.001$ \\
\hline DBP (mmHg) & $74 \pm 9$ & $70 \pm 9$ & $74 \pm 9$ & $75 \pm 9$ & $77 \pm 9$ & $<0.001$ \\
\hline $\mathrm{Cr}(\mathrm{mg} / \mathrm{dL})$ & $0.75 \pm 0.14$ & $0.65 \pm 0.13$ & $0.77 \pm 0.11$ & $0.80 \pm 0.11$ & $0.83 \pm 0.11$ & $<0.001$ \\
\hline eGFR $\left(\mathrm{mL} / \mathrm{min} / 1.73 \mathrm{~m}^{2}\right)$ & $91.2 \pm 17.6$ & $96.5 \pm 19.5$ & $91.1 \pm 16.6$ & $88.1 \pm 15.4$ & $84.3 \pm 15.0$ & $<0.001$ \\
\hline TC (mg/dL) & $192 \pm 29$ & $187 \pm 29$ & $192 \pm 29$ & $194 \pm 28$ & $201 \pm 28$ & $<0.001$ \\
\hline TG (mg/dL) & $94 \pm 59$ & $72 \pm 42$ & $91 \pm 51$ & $106 \pm 65$ & $126 \pm 76$ & $<0.001$ \\
\hline HDLC (mg/dL) & $64 \pm 15$ & $69 \pm 15$ & $64 \pm 15$ & $61 \pm 14$ & $59 \pm 16$ & $<0.001$ \\
\hline LDLC (mg/dL) & $113 \pm 26$ & $106 \pm 25$ & $113 \pm 26$ & $117 \pm 26$ & $122 \pm 27$ & $<0.001$ \\
\hline PG (mg/dL) & $93 \pm 10$ & $91 \pm 9$ & $93 \pm 10$ & $94 \pm 10$ & $96 \pm 11$ & $<0.001$ \\
\hline HbA1c (NGSP) (\%) & $5.23 \pm 0.34$ & $5.21 \pm 0.33$ & $5.23 \pm 0.33$ & $5.25 \pm 0.34$ & $5.29 \pm 0.37$ & $<0.001$ \\
\hline
\end{tabular}

$U A$ serum uric acid concentration, $B M I$ body mass index, $S B P$ systolic blood pressure, $D B P$ diastolic blood pressure, $C r$ serum creatinine concentration, $e G F R$ estimated glomerular filtration rate, $T C$ total cholesterol, $T G$ triglycerides, $H D L C$ high-density lipoprotein cholesterol, $L D L C$ low-density lipoprotein cholesterol, $P G$ plasma glucose concentration, HbAlc glycated hemoglobin 


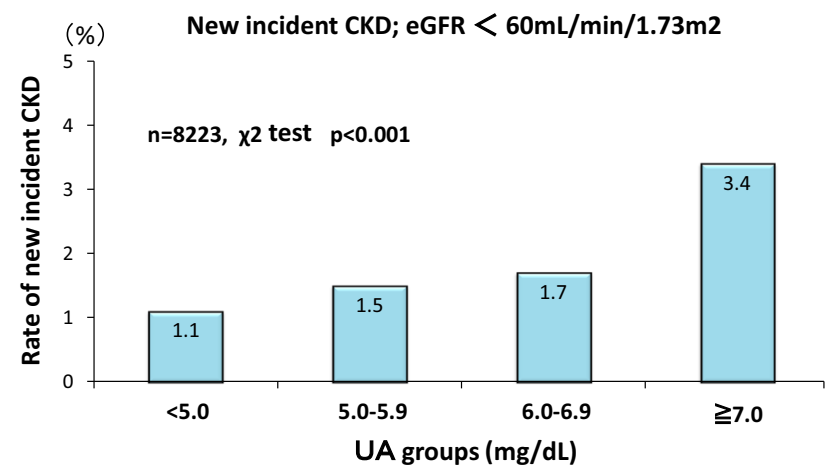

Fig. 1 New incidence of CKD at year 4. Newly onset of CKD was depicted at year 4 according to UA groups. The incidence rate increases significantly as UA levels increases $(p<0.001$ by the Chisquare analysis)

$5.7 \pm 1.4 \mathrm{mg} / \mathrm{dL}$, after the exclusion at the start, and the follow-up term was 4 years. Participants were all native Japanese. Greater UA levels were significantly associated with older age, males, greater prevalence of hypertension, BMI, waist circumference, SBP, DBP, serum $\mathrm{Cr}$ concentration, TC, TG, HDLC, LDLC, PG, HbA1c, and lesser eGFR (Table 1).

\section{Incidence of $C K D$}

Figure 1 showed the incident rates of newly developed CKD in a period of 4 years. Higher UA levels were significantly associated with greater incidence of CKD, $1.1 \%$ $(\mathrm{UA}<5.0 \mathrm{mg} / \mathrm{dL}), \quad 1.5 \% \quad(5.0-5.9 \mathrm{mg} / \mathrm{dL}), \quad 1.7 \%$ $(6.0-6.9 \mathrm{mg} / \mathrm{dL})$, and $3.4 \%(\geqq 7.0 \mathrm{mg} / \mathrm{dL})$, respectively (by the Chi-square test, $p<0.001$ ). The greater UA levels are associated with the higher incidence of CKD. This increasing trend is especially prominent in the individuals group with the highest UA level (UA $\geqq 7 \mathrm{mg} / \mathrm{dL}$ ). A total of 139 individuals had a decrease in eGFR less than $60 \mathrm{~mL} / \mathrm{min} / 1.73 \mathrm{~m}^{2}$ at year $4(139 / 8223$ individuals $=$ $1.7 \%)$. Among them, the rate of proteinuria at year 4 was $1.4 \%$ (2/139 individuals).

\section{Association of CKD onset with independent variables}

Table 2 showed various parameters that were associated the incidence of CKD, defined as a decline in eGFR less than $60 \mathrm{~mL} / \mathrm{min} / 1.73 \mathrm{~m}^{2}$ at year 4 . The Cox proportional hazard analysis shows that the estimates were eGFR [Hazard Ratio (HR) 0.816, $95 \%$ confidence intervals (CI) $0.791-0.840$ ] and male gender (HR 0.562, $95 \%$ CI $0.322-0.982$ ), suggesting that UA is not chosen as an estimate to predict the incident CKD in this multivariate analysis.
Table 2 Cox proportional hazard analysis to estimate the CKD onset

\begin{tabular}{lrlllr}
\hline Variable & \multicolumn{1}{l}{$\beta$} & SE $(\beta)$ & HR & $95 \%$ CI & \multicolumn{1}{l}{$p$} \\
\hline eGFR & -0.204 & 0.015 & 0.816 & $0.791-0.840$ & $<0.001$ \\
Male & -0.576 & 0.285 & 0.562 & $0.322-0.982$ & 0.043 \\
SBP & 0.021 & 0.011 & 1.021 & $0.999-1.043$ & 0.058 \\
DBP & -0.023 & 0.016 & 0.977 & $0.947-1.008$ & 0.151 \\
Age & 0.019 & 0.014 & 1.019 & $0.991-1.047$ & 0.187 \\
TC & -0.004 & 0.004 & 0.996 & $0.989-1.002$ & 0.202 \\
TG & 0.002 & 0.002 & 1.002 & $0.999-1.004$ & 0.307 \\
UA & 0.080 & 0.090 & 1.083 & $0.909-1.290$ & 0.375 \\
HbA1c & 0.141 & 0.289 & 1.152 & $0.654-2.027$ & 0.624 \\
BMI & 0.014 & 0.035 & 1.014 & $0.947-1.087$ & 0.686 \\
\hline Cox propor
\end{tabular}

Cox proportional hazard analysis was employed to identify factors to explain the onset of CKD

\section{Longitudinal outcome of CKD onset}

In 139 individuals with CKD at year 4, mean eGFR at baseline and closeout were $67.7 \pm 7.2$ and $57.2 \pm 2.5 \mathrm{~mL} /$ $\min / 1.73 \mathrm{~m}^{2}$, respectively. In contrast, mean eGFR of individuals without CKD incidence at baseline and closeout were $91.6 \pm 17.5$ and $84.4 \pm 13.7 \mathrm{~mL} / \mathrm{min} / 1.73 \mathrm{~m}^{2}$ $(n=8084)$, respectively.

\section{Hypertension analysis}

\section{Baseline characteristics}

Participants with hypertension at the start were excluded from the population for CKD analysis, eliminating 654 participants from 8223 , leaving 7569 participants for the hypertension analysis. This study group had an average age $39 \pm 10$ years, UA level $5.6 \pm 1.3 \mathrm{mg} / \mathrm{dL}$ at the start. Greater UA levels were associated with older age, males, greater BMI, waist circumference, SBP, DBP, serum $\mathrm{Cr}$ concentration, TC, TG, HDLC, LDLC, PG, HbA1c, and lesser eGFR (Table 3).

\section{Incidence of newly-developed hypertension}

Figure 2 showed the incident rates of newly developed hypertension within a period of 4 years. Higher UA levels had a close association with the new hypertension; $5.0 \%$ $(\mathrm{UA}<5.0 \mathrm{mg} / \mathrm{dL}), \quad 8.9 \% \quad(5.0-5.9 \mathrm{mg} / \mathrm{dL}), \quad 10.6 \%$ $(6.0-6.9 \mathrm{mg} / \mathrm{dL})$, and $11.8 \%(\geqq 7.0 \mathrm{mg} / \mathrm{dL})$, respectively ( $p<0.001$ by the Chi-square test). The greater incidence remained linearly associated with increasing UA level. A total of 633 individuals developed hypertension (633/7569 individuals $=8.4 \%$ ). Among them, the rate of proteinuria at year 4 was $1.6 \%$ (10/633 individuals). In addition, among 633 individuals who became hypertensives, there 
Table 3 Demographics of subjects for hypertension development

\begin{tabular}{|c|c|c|c|c|c|c|}
\hline & \multicolumn{6}{|c|}{ Uric acid groups (mg/dL) } \\
\hline & Overall & $<5.0$ & $5.0-5.9$ & $6.0-6.9$ & $\geqq 7.0$ & $p$ \\
\hline$N$ & 7569 & 2531 & 2247 & 1894 & 897 & \\
\hline Age (years) & $39 \pm 10$ & $37 \pm 9$ & $39 \pm 10$ & $40 \pm 9$ & $41 \pm 9$ & $<0.001$ \\
\hline $\operatorname{Men}(\%)$ & 76.3 & 40.9 & 89.2 & 97.8 & 98.8 & $<0.001$ \\
\hline BMI $\left(\mathrm{Kg} / \mathrm{m}^{2}\right)$ & $22 \pm 3$ & $21 \pm 3$ & $22 \pm 3$ & $23 \pm 3$ & $24 \pm 3$ & $<0.001$ \\
\hline Waist circumference $(\mathrm{cm})$ & $78.1 \pm 8.6$ & $73.9 \pm 8.1$ & $78.3 \pm 7.8$ & $80.8 \pm 7.8$ & $83.7 \pm 8.1$ & $<0.001$ \\
\hline $\mathrm{SBP}(\mathrm{mmHg})$ & $115 \pm 11$ & $112 \pm 11$ & $116 \pm 11$ & $117 \pm 10$ & $119 \pm 10$ & $<0.001$ \\
\hline DBP (mmHg) & $72 \pm 8$ & $70 \pm 8$ & $73 \pm 8$ & $74 \pm 8$ & $75 \pm 7$ & $<0.001$ \\
\hline $\mathrm{Cr}(\mathrm{mg} / \mathrm{dL})$ & $0.75 \pm 0.14$ & $0.65 \pm 0.13$ & $0.77 \pm 0.11$ & $0.80 \pm 0.11$ & $0.83 \pm 0.11$ & $<0.001$ \\
\hline eGFR $\left(\mathrm{mL} / \mathrm{min} / 1.73 \mathrm{~m}^{2}\right)$ & $91.4 \pm 17.7$ & $96.5 \pm 19.6$ & $91.2 \pm 16.7$ & $88.1 \pm 15.3$ & $84.1 \pm 15.0$ & $<0.001$ \\
\hline $\mathrm{TC}(\mathrm{mg} / \mathrm{dL})$ & $191 \pm 29$ & $187 \pm 28$ & $191 \pm 29$ & $194 \pm 28$ & $201 \pm 29$ & $<0.001$ \\
\hline $\mathrm{TG}(\mathrm{mg} / \mathrm{dL})$ & $91 \pm 57$ & $71 \pm 41$ & $91 \pm 51$ & $104 \pm 62$ & $125 \pm 76$ & $<0.001$ \\
\hline HDLC (mg/dL) & $64 \pm 15$ & $69 \pm 15$ & $63 \pm 15$ & $61 \pm 14$ & $59 \pm 15$ & $<0.001$ \\
\hline LDLC (mg/dL) & $112 \pm 26$ & $106 \pm 25$ & $113 \pm 26$ & $116 \pm 26$ & $122 \pm 27$ & $<0.001$ \\
\hline PG (mg/dL) & $93 \pm 9$ & $91 \pm 9$ & $93 \pm 10$ & $94 \pm 9$ & $96 \pm 10$ & $<0.001$ \\
\hline HbA1c (NGSP) (\%) & $5.23 \pm 0.34$ & $5.20 \pm 0.33$ & $5.23 \pm 0.32$ & $5.25 \pm 0.34$ & $5.29 \pm 0.37$ & $<0.001$ \\
\hline
\end{tabular}

$U A$ serum uric acid concentration, $B M I$ body mass index, $S B P$ systolic blood pressure, $D B P$ diastolic blood pressure, $C r$ serum creatinine concentration, $e G F R$ estimated glomerular filtration rate, $T C$ total cholesterol, $T G$ triglycerides, $H D L C$ high-density lipoprotein cholesterol, $L D L C$ low-density lipoprotein cholesterol, $P G$ plasma glucose concentration, $H b A 1 c$ glycated hemoglobin

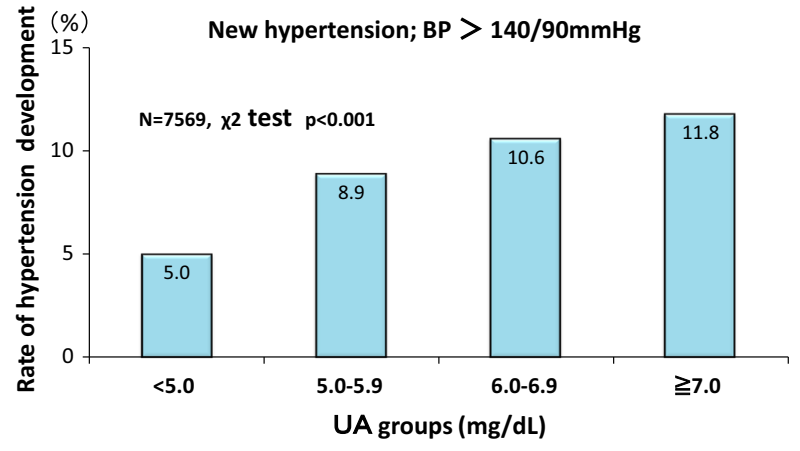

Fig. 2 New development of hypertension at year 4. New onset of hypertension was depicted at year 4 according to UA groups. The incidence rate increases significantly as UA levels increases $(p<0.001$ by the Chi-square analysis $)$

were 13 individuals who developed both CKD and hypertension at year 4 (data not shown).

\section{Association of hypertension onset with independent variables}

Table 4 showed various parameters that were associated with the onset of hypertension defined as a rise of SBP to $140 \mathrm{mmHg}$ or greater, DBP of $90 \mathrm{mmHg}$ or greater at year 4. Cox proportional hazard analysis shows that such variables were BMI (HR 1.190, 95 \% CI 1.155-1.226), age
Table 4 Cox proportional hazard analysis to estimate the hypertension development

\begin{tabular}{lrllll}
\hline Variable & \multicolumn{1}{l}{$\beta$} & $\mathrm{SE}(\beta)$ & HR & $95 \%$ CI & $p$ \\
\hline BMI & 0.174 & 0.015 & 1.190 & $1.155-1.226$ & $<0.001$ \\
Age & 0.021 & 0.006 & 1.021 & $1.010-1.032$ & $<0.001$ \\
HDLC & 0.014 & 0.003 & 1.013 & $1.007-1.019$ & $<0.001$ \\
Male & 0.583 & 0.149 & 1.791 & $1.338-2.395$ & $<0.001$ \\
UA & 0.106 & 0.042 & 1.112 & $1.024-1.207$ & 0.012 \\
eGFR & 0.008 & 0.003 & 1.008 & $1.002-1.013$ & 0.012 \\
TG & 0.001 & 0.001 & 1.000 & $0.999-1.002$ & 0.319 \\
HbA1c & -0.053 & 0.147 & 0.948 & $0.711-1.265$ & 0.718 \\
\hline Cox proport & & & & &
\end{tabular}

Cox proportional hazard analysis was employed to identify factors to explain the onset of CKD. BMI, Age, Sex, HDLC, and UA were chosen as such independent variables

(HR 1.021, $95 \%$ CI 1.010-1.032), HDL-cholesterol (HR 1.013, $95 \%$ CI 1.007-1.019), male gender (HR 1.791, $95 \%$ CI 1.338-2.395), UA level (HR 1.112, $95 \%$ CI 1.024-1.207), and eGFR (HR 1.008, $95 \%$ CI 1.002-1.013).

\section{Longitudinal relationship between UA groups and hypertension}

In 633 individuals with hypertension at year 4 , the $\mathrm{BP}$ at baseline and closeout were $124.2 \pm 8.4 \mathrm{mmHg}$ in SBP, 
Table 5 Logistic regression analysis to estimate the hypertension development

\begin{tabular}{|c|c|c|c|c|}
\hline \multicolumn{5}{|l|}{ Uric acid groups $(\mathrm{mg} / \mathrm{dL})$} \\
\hline & $<5.0$ & $5.0-5.9$ & $6.0-6.9$ & $\geqq 7.0$ \\
\hline Development of hypertension $(n=633 ; 8.4 \%)$ & $127(20.1 \%)$ & $199(31.4 \%)$ & $201(31.8 \%)$ & $106(16.7 \%)$ \\
\hline Unadjusted OR $(95 \% \mathrm{CI})$ & 1.00 (reference) & $1.84(1.46-2.32)$ & $2.25(1.78-2.83)$ & $2.54(1.94-3.32)$ \\
\hline Adjusted OR (95 \%CI) & 1.00 (reference) & $1.30(1.00-1.69)$ & $1.38(1.05-1.81)$ & $1.33(1.01-1.80)$ \\
\hline
\end{tabular}

The development of hypertension was defined as a BP rise more than 140/90 $\mathrm{mmHg}$ in systolic and/or diastolic at year 4 . The OR was adjusted for age, sex, BMI, waist circumference, SBP, DBP, TC, TG, HDLC, LDLC, PG, and HbA1c

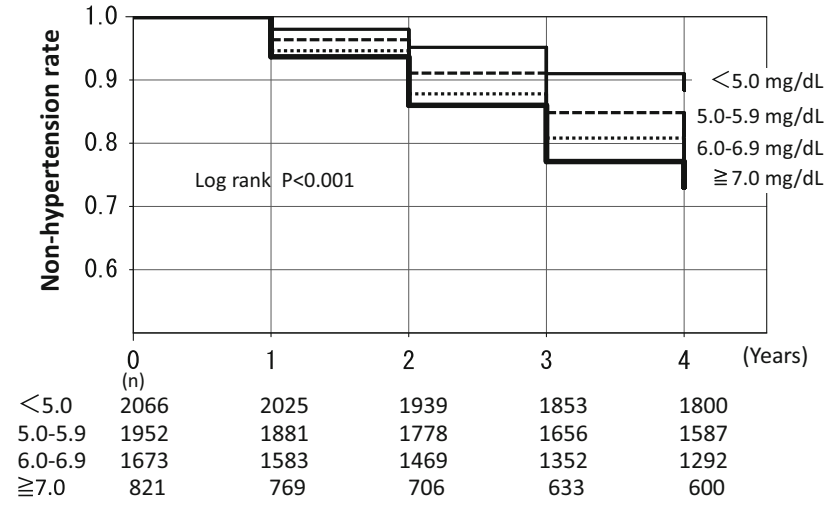

Fig. 3 Hypertension-free rate according to UA groups. The occurrence of hypertension in 4 years was presented according to UA groups. Each line has proven statistically different by the Log-rank test $(p<0.001)$

$78.3 \pm 6.7 \mathrm{mmHg}$ in $\mathrm{DBP}$ and $140.7 \pm 10.0 \mathrm{mmHg}$ in SBP, $91.6 \pm 7.1 \mathrm{mmHg}$ in DBP, respectively. Mean eGFR at baseline and closeout were $89.5 \pm 16.9$ and $83.5 \pm 13.7 \mathrm{~mL} / \mathrm{min} / 1.73 \mathrm{~m}^{2}$, respectively. In contrast, mean eGFR of individuals without developing hypertension within 4 years $(n=6936)$ at baseline and closeout were $91.5 \pm 17.8$ and $84.1 \pm 14.1 \mathrm{~mL} / \mathrm{min} / 1.73 \mathrm{~m}^{2}$, respectively. Greater UA levels were associated with greater OR of hypertension development. After adjustment for age, sex, BMI, waist circumference, SBP, DBP, TC, TG, HDLC, LDLC, PG, and HbA1c, multivariate adjusted OR of the logistic regression analysis for new onset of hypertension was 1.00 (reference), 1.30 (95\% CI 1.00-1.69), 1.38 (95\% CI 1.05-1.81), and 1.33 (95\% CI 1.01-1.80) for Group 1 through Group 4, respectively (Table 5). All three groups of UA levels (Group 2, 3, and 4) remained significantly associated with newly developed hypertension.

\section{Association between hypertension development and UA}

Figure 3 showed Kaplan-Meier analysis to indicate hypertension-free rates according to UA groups. The survival from hypertension at year 4 was calculated as a function of each UA group. There was a statistical difference in the hypertension-free rate among the 4 groups with different
UA levels (the Log-rank test, $p<0.001$ ). Furthermore, multivariate Cox proportional hazard analysis shows that the UA levels to be associated with hypertension development were Group 2 (UA: $5.0-5.9 \mathrm{mg} / \mathrm{dL}$, HR 1.382, $95 \%$ CI 1.152-1.658), Group 3 (UA: $6.0-6.9 \mathrm{mg} / \mathrm{dL}$, HR $1.673,95 \%$ CI 1.389-1.658), and Group 4 (UA $\geqq 7.0 \mathrm{mg} / \mathrm{dL}$, HR 2.006, $95 \%$ CI 1.634-2.463) as a reference of Group 1 (UA $<5.0 \mathrm{mg} / \mathrm{dL}$ ).

\section{Discussion}

The present study demonstrates that UA levels were associated with the development of hypertension, but not with the incidence of CKD. The strengths of this study are the large number of individuals who did not have CKD or hypertension at the entry, the complete nature of the data set, and the ability to link demographic and clinical factors with CKD and rise in BP. Other advantages include no new medications for hyperuricemia, diabetes, hypertension, and dyslipidemia throughout the observation period and the ability to adjust for multiple factors that may affect initiation of CKD and hypertension. We also controlled for the currently treated diabetes, hypertension, hyperuricemia, dyslipidemia, and MACE etc., all known as well-established predictors of kidney dysfunction.

\section{Association of UA levels with CKD incidence}

Increased UA is associated with increased risk for future hypertension, diabetic nephropathy, and CKD, and that hyperuricemia, per se, could be an independent risk factor for poor cardiovascular prognosis [1-8, 16, 17]. Advanced CKD frequently induces fatal cardiovascular complications [12]. UA level is an independent risk factor for the progression of kidney disease in a variety of kidney diseases $[5-9,16,17]$. In addition, a prospective controlled trial examined the clinical effect of decreasing UA level with allopurinol in patients with CKD and hyperuricemia [18]. The study disclosed that subjects in the UA-lowering group showed less kidney dysfunction, suggesting a role of UA in the pathogenesis of CKD. 
The cause of CKD is multiple. Thus, it is of interest to look into factors that have impact on both the initiation and the progression of CKD in conjunction with UA levels. So far, the role of UA on the incidence of CKD is controversial [12-14, 18-21]. Weiner et al. [20] recruited 13,338 participants with intact kidney function in two communitybased cohorts and concluded that elevated UA level is a modest, independent risk factor for incident CKD. Furthermore, a meta-analysis consisting of 15 cohorts with a recruitment of 99,205 individuals and 3492 incident CKD cases showed that the relative risk of CKD was $1.22(95 \%$ CI 1.16-1.28) per $1 \mathrm{mg} / \mathrm{dL}$ serum UA level increment, suggesting a positive link between the two [22].

The present study demonstrates that after the adjustment of confounding factors by the multivariate analysis, UA is no longer an estimate to predict the incident CKD (Table 2), despite the positive link between the incidence rates and the levels of UA (Fig. 1). It is apparent that UA levels are greatly influenced by the confounding effect of eGFR. The involvement of UA on the CKD incidence, in this context, could be difficult to detect in an epidemiological study. We can conclude, at this point, that high UA does not act as a trigger for new incidence of CKD.

\section{Association of UA levels with hypertension development}

Animal experiments demonstrate that increased serum UA causes hypertension that is reversible at the early stage but becomes irreversible at the later stage [9]. In human, substantial number of literature reviews shows a dose-dependent, linear consistent relationship of UA level with BP [23-29]. A total of 18 prospective cohort studies representing data from 55,607 participants clearly demonstrate that hyperuricemia was associated with an increased risk for incident hypertension (adjusted relative ratio, 1.41, $95 \%$ CI 1.23-1.58). These effects were significantly larger in younger populations and tended to be larger in women, and the effect may be larger among African American individuals, concluding that hyperuricemia is associated with an increased risk for incident hypertension, independent of traditional hypertension risk factors [25]. Similarly, a recent meta-analysis consisting of 25 studies with a recruitment of 97,824 individuals showed a modest link between hyperuricemia and incident hypertension suggesting that hyperuricemia was associated with a higher risk of incident hypertension [30].

Our present study confirms previous studies that hyperuricemia, per se, worsened hypertension but also act as a trigger in its first onset. There were a small number of overlapping participants (13 individuals) who developed both CKD and hypertension at year 4 , but we feel that this concomitance might not affect the positive link between the development hypertension and UA level.

A prospective cohort study recruiting 49,413 Japanese workers with a 7-year follow-up showed hyperuricemia has a strong association with the risks of death in all causes, CHD, stroke, liver disease and CKD suggesting that high UA appeared to be a considerable risk factor for reduced life expectancy [31].

\section{Conclusions}

The present trial shows that high UA levels are associated with the new development of hypertension, but not with the incidence of CKD. Whether lowering of UA levels in hyperuricemic patients is beneficial or not remains to be investigated in the future.

Acknowledgments We are indebted to Ms. Tomoko Tanabe for her technical expertise on the statistical analyses.

Conflict of interest The authors declare no conflict of interest.

Open Access This article is distributed under the terms of the Creative Commons Attribution 4.0 International License (http:// creativecommons.org/licenses/by/4.0/), which permits unrestricted use, distribution, and reproduction in any medium, provided you give appropriate credit to the original author(s) and the source, provide a link to the Creative Commons license, and indicate if changes were made.

\section{References}

1. Jossa F, Farinaro E, Panico S, Krogh V, Celentano E, Galasso R, Mancini M, Trevisan M. Serum uric acid and hypertension: the Olivetti Heart Study. J Hum Hypertens. 1994;8:677-81.

2. Fang J, Alderman MH. Serum uric acid and cardiovascular mortality: the NHANES I epidemiologic follow-up study, 1971-1992. National Health and Nutrition Examination Survey. JAMA. 2000;283:2404-10.

3. Mellen PB, Bleyer AJ, Erlinger TP, Evans GW, Nieto FJ, Wagenknecht LE, Wofford MR, Herrington DM. Serum uric acid predicts incident hypertension in a biethnic cohort: the atherosclerosis risk in communities study. Hypertension. 2006;48(6): 1037-42.

4. Krishnan E, Kwoh CK, Schumacher HR, Kuller L. Hyperuricemia and incidence of hypertension among men without metabolic syndrome. Hypertension. 2007;49(2):298-303.

5. Taniguchi Y, Hayashi T, Tsumura K, Endo G, Fujii S, Okada K. Serum uric acid and the risk for hypertension and Type 2 diabetes in Japanese men. The Osaka Health Survey. J Hypertens. 2001;19(7):1209-15.

6. Kawai T, Ohishi M, Takeya Y, Onishi M, Ito N, Yamamoto K, Kamide K, Rakugi H. Serum uric acid is an independent risk factor for cardiovascular disease and mortality in hypertensive patients. Hypertens Res. 2012;35(11):1087-92.

7. Syrjanen J, Mustonen J, Pasternack A. Hypertriglyceridaemia and hyperuricaemia are risk factors for progression of $\operatorname{IgA}$ nephropathy. Nephrol Dial Transplant. 2000;15:34-42. 
8. Bo S, Cavallo-Perin P, Gentile L, Repetti E, Pagano G. Hypouricemia and hyperuricemia in type 2 diabetes: two different phenotypes. Eur J Clin Invest. 2001;31:318-21.

9. Fessel WJ. Renal outcomes of gout and hyperuricemia. Am J Med. 1979;67:74-82.

10. Feig DI, Kang DH, Johnson RJ. Uric acid and cardiovascular risk. N Engl J Med. 2008;359(17):1811-21.

11. Yu TF, Berger L. Impaired renal function gout: its association with hypertensive vascular disease and intrinsic renal disease. Am J Med. 1982;72:95-100.

12. Johnson RJ, Segal MS, Srinivas T, Ejaz A, Mu W, Roncal C, Sánchez-Lozada LG, Gersch M, Rodriguez-Iturbe B, Kang DH, Acosta JH. Essential hypertension, progressive renal disease, and uric acid: a pathogenetic link? J Am Soc Nephrol. 2005;16:1909-19.

13. Kanellis J, Feig DI, Johnson RJ. Does asymptomatic hyperuricaemia contribute to the development of renal and cardiovascular disease? An old controversy renewed. Nephrology (Carlton). 2004;9:394-9.

14. Zhang W, Sun K, Yang Y, Zhang H, Hu FB, Hui R. Plasma uric acid and hypertension in a Chinese community: prospective study and meta-analysis. Clin Chem. 2009;55(11):2026-34.

15. Matsuo S, Imai E, Horio M, Yasuda Y, Tomita K, Nitta K, Yamagata K, Tomino Y, Yokoyama H, Hishida A. On behalf of the collaborators developing the Japanese equation for estimated GFR. Revised equations for estimated GFR from serum creatinine in Japan. Am J Kidney Dis. 2009;53:982-92.

16. Iseki K, Oshiro S, Tozawa M, Iseki C, Ikemiya Y, Takishita S. Significance of hyperuricemia on the early detection of renal failure in a cohort of screened subjects. Hypertens Res. 2001;24:691-7.

17. Iseki $\mathrm{K}$, Ikemiya $\mathrm{Y}$, Inoue $\mathrm{T}$, Iseki $\mathrm{C}$, Kinjo $\mathrm{K}$, Takishita $\mathrm{S}$. Significance of hyperuricemia as a risk factor for developing ESRD in a screened cohort. Am J Kidney Dis. 2004;44:642-50.

18. Siu YP, Leung KT, Tong MK, Kwan TH. Use of allopurinol in slowing the progression of renal disease through its ability to lower serum uric acid level. Am J Kidney Dis. 2006;47:51-9.

19. Chonchol M, Shlipak MG, Katz R, Sarnak MJ, Newman AB, Siscovick DS, Kestenbaum B, Carney JK, Fried LF. Relationship of uric acid with progression of kidney disease. Am J Kidney Dis. 2007;50:239-47.

20. Werner DE, Tighiouart H, Elsayed EF, Griffith JL, Salem DN, Levey AS. Uric acid and incident kidney disease in the community. J Am Soc Nephrol. 2008;19:1204-11.
21. Zhu P, Liu Y, Han L, Xu G, Ran JM. Serum uric acid is associated with incident chronic kidney disease in middle-aged populations: a meta-analysis of 15 cohort studies. PLoS One. 2014;9(6):e100801. doi:10.1371/journal.pone.0100801 eCollection 2014.

22. Go AS, Chertow GM, Fan D, McCulloch CE, Hsu CY. Chronic kidney disease and the risks of death, cardiovascular events, and hospitalization. N Engl J Med. 2004;351(13):1296-305.

23. Johnson RJ, Feig DI, Kang DH, Herrera-Acosta J. Resurrection of uric acid as a causal risk factor for essential hypertension. Hypertension. 2005;45:18-20.

24. Nakagawa T, Kang DH, Feig D, Sanchez-Lozada LG, Srinivas TR, Sautin Y, Ejaz AA, Segal M, Johnson RJ. Unearthing uricacid: an ancient factor with recently found significance in renal and cardiovascular disease. Kidney Int. 2006;69(10):1722-5.

25. Grayson PC, Kim SY, LaValley M, Choi HK. Hyperuricemia and incident hypertension: a systematic review and meta-analysis. Arthritis Care Res. 2011;63(1):102-10.

26. Cannon PJ, Stason WB, Demartini FE, Sommers SC, Laragh JH. Hyperuricemia in primary and renal hypertension. N Engl J Med. 1996;275:457-64.

27. Kinsey D, Walther R, Sise HS, Whitelaw G, Smithwick R. Incidence of hyperuricemia in 400 hypertensive subjects. Circulation. 1961;24:972-3.

28. Klein R, Klein BE, Cornoni JC, Maready J, Cassel JC, Tyroler HA. Serum uric acid. Its relationship to coronary heart disease risk factors and cardiovascular disease, Evans County, Georgia. Arch Intern Med. 1973;132:401-10.

29. Feig DI, Mazzali M, Kang DH, Nakagawa T, Price K, Kannelis J, Johnson RJ. Serum uric acid: a risk factor and a target for treatment? J Am Soc Nephrol. 2006;17 (suppl 2):S69-73.

30. Wang J, Qin T, Chen J, Li Y, Wang L, Huang H, Li J. Hyperuricemia and risk of incident hypertension: a systematic review and meta-analysis of observational studies. PLoS One. 2014;9(12):e114259. doi:10.1371/journal.pone.0114259 eCollection 2014.

31. Tomita M, Mizuno S, Yamanaka H, Hosoda Y, Sakuma K, Matsuoka Y, Okada M, Yamaguchi M, Yosida H, Morisawa, Murayama T. Does hyperuricemia affect mortality? A prospective cohort study of Japanese male workers. J Epidemiol. 2000;10:403-9. 